\title{
An investigation into the chronic effects of flavonoids in orange juice on cardiovascular health and cognition
}

\author{
R. J. Kean ${ }^{1,2}$, J. Freeman ${ }^{1}$, J. A. Ellis ${ }^{1}$, L. T. Butler ${ }^{1}$ and J. P. E. Spencer ${ }^{2}$ \\ ${ }^{1}$ Department of Food and Nutritional Sciences, University of Reading, Reading RG6 6UR, ${ }^{2}$ School of Psychology and \\ Clinical Language Sciences, University of Reading, PO Box 238, Reading, RG6 6AL, UK
}

Flavonoids are a diverse class of natural compounds, ubiquitous in plants. They are abundant within the human diet and in recent years there has been increasing interest in the potential health benefits associated with the consumption of flavonoid-containing foods, including possible benefits for cognitive function. Studies have shown that flavonoids can result in anti-oxidant ${ }^{(1)}$, anti-inflammatory ${ }^{(2)}$ and neuroprotective $^{(3)}$ effects and dietary intervention studies in animals have demonstrated beneficial effects on vascular function and cognitive performance ${ }^{(4)}$. Although the available data may provide considerable justification for recommending increased flavonoid intake by the population as a public health strategy to reduce the population burden of age-related cognitive decline, there is a distinct paucity of information from randomised controlled trials demonstrating their bio-efficiency in humans.

We employed a randomized, double blind, cross over design in which 37 healthy older adults (60-82 years) consumed $500 \mathrm{ml}$ of experimental orange juice $(549 \mathrm{mg} / \mathrm{L}$ hesperidin, $60 \mathrm{mg} / \mathrm{L}$ narirutin) or control juice $(64 \mathrm{mg} / \mathrm{L}$ hesperidin, $10 \mathrm{mg} / \mathrm{L}$ narirutin) daily for 8 weeks, with a 4 week wash out between conditions. All blood pressure measurements were collected from 12 hour fasted volunteers before and after the eight week treatment periods. Cognitive measures of Processing Speed (Digit Symbol Substitution Task) and Delayed Word Recall (CERAD) were measured after a standardised, low polyphenol breakfast.

For blood pressure (BP) analysis participants were grouped by Systolic BP (SBP) at baseline with $<135 \mathrm{mmHg}$ indicating low group and $>136 \mathrm{mmHg}$ indicating high group. There were no significant differences in SBP pre and post the 8 week treatment, in either group, for the control orange juice condition. For the study juice, the Low group showed an increase in SBP $(p=.05)$ and the High group showed a significant decrease in SBP $(p=.044)$. The cognitive measures also yielded interesting results with a significant improvement in processing speed of the Digit Symbol Substitution Task in the study condition $(p=.049)$ and not in the control juice condition $(p>.05)$ and a significant improvement in the Delayed Recall task in the experimental $(p=.009)$ but not control $(p>.05)$ condition.

This study indicates that hesperidin, its major flavonoid, could be causally linked to the beneficial effect of orange juice on cardiovascular health and supports previous findings that long term consumption of orange juice can reduce blood pressure ${ }^{(5)}$. Also that regular consumption of high flavonoid orange juice could help attenuate the cognitive decline seen in older adulthood. These findings imply that a high flavonoid diet can help preserve encoding of information (Processing Speed) and long term recall of information (Delayed Recall) which replicate reports from animal studies of high polyphenol diet having a beneficial effect on cognition in late adulthood ${ }^{(4)}$ and provides justification for recommending increased flavonoid intake by the population as a public health strategy to reduce the population burden of age-related cognitive decline.

1. Prior RL. (2003) Am J Clin Nutr 78(3): 570S-578S

2. Gonzalez-Gallego J, Sanchez-Campos S \& Tunon MJ (2007) Nutr Hosp 22(3): 287-293.

3. Fisher NDL, Hughes M, Gerhard-Herman et al. (2003) Hypertension 21(12): 2281-2286.

4. Williams RJ, Spencer JP \& Rice-Evans C. (2004) Free Radic Bio. Med 36, 838-84.

5. Morand C, Dubray C, Milenkovic D et al. (2011) Am J Clin Nutr 93, 73-80. 